\title{
Mit E-DOC Gesundheitskosten wieder im Griff!
}

Max Konzelmann

Korrespondenz:

Dr. med. Max Konzelmann

Asylstrasse 39

CH-8750 Glarus

max.konzelmann@yahoo.de
Die bisherigen Bemühungen zur Reduktion der Gesundheitskosten bzw. der Krankenkassenprämien haben versagt. Weder die Senkung der Medikamentenkosten, noch die Couchepin'sche Zugangsgebühr zur Arztpraxis haben eine Chance. Dasselbe gilt für die generelle Franchise-Erhöhung oder das ebenso asoziale Bonus-Malus-System.

In Anbetracht des aufgeblähten Gesundheitsmarktes können nur innovative und vernetzte Systeme zum Ziel führen. Hier kommt SPARMED mit dem ausgereiften Projekt E-DOC zum Zuge. E-DOC entlastet Tausende von überbeanspruchten Ärzten und wird den heutigen Ärztemangel sukzessive beheben. Der Patient muss sich nicht mehr um einen Arzttermin bemühen. Im Internet klickt er auf GAGELMED, wählt im Browser den SYMPTOMATOR, gibt die Symptome ein und kann im E-DIAGNOST sehen, woran er leidet. Für die Behandlung geht er anschliessend auf THERAPORT, worauf Therapieanweisung und Rezepte automatisch ausgedruckt werden. So kann er ohne unnötigen Arztbesuch zur Apotheke, wenn er die Medikamente nicht von APO-MAIL DIRECT schicken lässt.

Ein Beispiel: Wegen Brennens beim Wasserlösen wählt der Patient GAGELMED. E-DIAGNOST meldet: «Blasenentzündung oder Gonorrhoe. Finden Sie in der Unterwäsche keine Flecken wie von Vanillecrème, haben Sie Blasenentzündung und klicken dieses Wort an. Das Rezept für Furadantin wird ausgedruckt, sofern Sie nicht Bärentraubentee, Blasosan oder Spagyrocyst vorziehen. In diesem Fall bitte «Altern〉 eingeben. Ist es aber Gonorrhoe, erhalten Sie das Rezept für Azithromycin.»

Das Beispiel dokumentiert die Überlegenheit von GAGELMED. Der Patient vergeudet keine Zeit im Wartezimmer und beim Arzt, bekommt eine objektive Information über seine Krankheit, erhält auch sofort das richtige Medikament und muss weniger Prämie bezahlen. Parallel zu E-DOC läuft die Rekrutierung der Mediaziner. Diese ärztliche Variante der Mediatiker ist nötig, um die Bevölkerung über Krankheiten und Behandlungsverfahren besser zu informieren, als das beim herkömmlichen Hausarzt oder Spezialisten der Fall ist.

Der ideale Mediaziner präsentiert sich in der täglichen DOC-Sendung einnehmend telegen, sollte aber von themaspezifischem Aussehen sein. Werden kompliziertere Krankheiten erläutert, wäre ein Mediaziner vom Typ des Stefan Klapproth willkommen. Geriatrische Fälle stellen Mediaziner vor, die über die Ausstrahlung von Cool Man Steiner, Pfarrer Sieber oder Stephanie Glaser verfügen. Wird am Bildschirm gezeigt, wie sich der Chirurg in den Eingeweiden des
Patienten mit magischen Händen vorarbeitet, das Herz eröffnet oder gar im Gehirn hantiert, dann ist mehr die Handfertigkeit als das Gesicht gefragt, das ohnehin steril abgedeckt ist. Die Mediazinerinnen oder Mediaziner, die belehrende Sendungen moderieren, in denen erklärt wird, was Hämorrhoiden sind und warum, oder weshalb Kopfschuppen auch bei mageren Menschen fettig sein können, und dass die Antibabypille fast nur bei Frauen sinnvoll eingesetzt werden kann, sollen nett und sympathisch aussehen. Da der Bedarf gross ist, werden die durch das E-DOC überzählig gewordenen Ärzte hier ihr Aus- und Einkommen finden.

\section{«Dem bahnbrechenden System SPARMED ist mit E-DOC zweifellos ein grosser Wurf gelungen»}

Wichtig ist ausser dem Aussehen die verbale Kommunikation. Der Mediaziner stellt die Patienten im Dialekt mitfühlend volksnah vor und lässt gekonnt Abkürzungen und lateinische Wörter einfliessen. Der Clochard mit Kreislauf- und Atemstörung wird wie folgt vorgestellt: «Heute zeige ich Ihnen diesen klassischen WINIWARTER-BÜRGER, dessen PAVK-bedingte Claudicatio bekanntlich wegen des vielen Rauchens entstanden ist, was ja auch für die COPD gilt.» Dann wendet er sich jovial dem Patienten zu: «So, Herr Karcher, zeigetsi de Lüüt emaal wiä Si da bim Fürelaufe de Chrampf im Bei überchömed. Und dänn tüemer au na chreftig huäschte, gäll, damit d'Zuäschauer ghööred, wiä's bi Ine amigs tönt.» Falls der Ärztemangel andauert, können Mediaziner aus Deutschland eingesetzt werden, wie z. B. Horst Tefauk aus Pommern, der Interessantes aus der Notfallstation berichtet: «Hier wollend mir üüch diese schöne Buuchmuskeleverstuchung zeige, wo gerade inegechoo ischt. Sie chönnend das guet an der verkrümmte Haltig vom Patient erluege. Dem Maa könned mir in üserem Krankehuus mit de physiotherapütische Methode hütte eini perfekte Behandligung aabüüte, wo ihn rasch heile tuet.»

Dem bahnbrechenden System SPARMED ist mit E-DOC zweifellos ein grosser Wurf gelungen. Die Gesundheitskosten sinken, der Ärztemangel wird behoben und dem Patienten steht trotzdem eine Medizin auf höchstem Niveau zur Verfügung. Eine weitere Verbilligung bei gleichzeitiger Verbesserung der Qualität wird in ca. 6 Monaten das System SPITOUT bringen. Die Fachleute vom BAG arbeiten Tag und Nacht daran. 